\title{
Effect of Ultrafast Cooling on Pore Formation in Amorphous Titanium Nickelide
}

\author{
B.N. GalimzYAnOV ${ }^{a, b, *}$, G.A. Nikiforov ${ }^{a}$ And A.V. MOKShin ${ }^{a, b}$ \\ ${ }^{a}$ Kazan Federal University, 420008 Kazan, Russia \\ ${ }^{b}$ Udmurt Federal Research Center of the Ural Branch of the Russian Academy of Sciences, 426068 Izhevsk, Russia
}

(Received December 24, 2019; revised version February 10, 2020; in final form February 12, 2020)

\begin{abstract}
Materials with amorphous porous structure are the perspective due to unique combination of their mechanical and energy-absorption properties. We perform atomistic dynamics simulations of amorphous titanium nickelide under isobaric conditions with pressure of $1 \mathrm{~atm}$. Effect of cooling at extremely high rates on the formation of pores in amorphous titanium nickelide is studied. For liquid phase, we determine a vaporization temperature and equation of states. We find that the pore morphology including the average linear size of pores depends on the magnitude of the cooling rate. The porosity of this amorphous solid does not depend on such high cooling rates. This finding is in good agreement with experimental and simulation results obtained for solidification of porous Al-based alloys. The results of the present work will be useful in study of mechanical properties of amorphous metal alloys with a nanoporous structure.
\end{abstract}

DOI: 10.12693/APhysPolA.137.1149

PACS/topics: amorphous titanium nickelide, mesoporous structure, cooling rate, structure, atomistic dynamics

\section{Introduction}

Porous titanium nickelide $\left(\mathrm{Ni}_{50} \mathrm{Ti}_{50}\right.$ alloy or $\left.\mathrm{NiTi}\right)$ is the most famous and widely functional material for many applications due to its unique mechanical properties, excellent corrosion resistance and biocompatibility [1-7]. These properties are largely determined by the specificity of the porous structure, such as the presence of percolating pore networks or isolated pores in the system, as well as the size and geometry of the pores. One of the significant achievements is synthesis of $\mathrm{Ni}$ and Ti-based metallic foams with micro- or nanosized pores, with low density, high strength, high surface area, and open porosity [8]. The main application of such foams with microsized pores is related with production of bone implants [9-11], whereas metallic foams with nanoporous structure are demanded, for example, in production of energy-storage elements [12, 13]. Moreover, in metallic foams the pore morphology (closed-cell or open-cell) plays a crucial role $[8,14]$. The porous structure may consist of interconnected and/or spatially separated cells, and the presence of such cells determines the mechanical properties of the porous systems and, eventually, the fields of their application.

Despite the successes achieved in the development of technologies for the production of porous metal alloys, the experimental and simulation studies of porous amorphous NiTi alloy are very poor. The production of porous amorphous NiTi alloy is much more complicated primarily due to the high melting point of titanium nickelide $\left(T_{m} \approx 1583 \mathrm{~K}\right)[1,2]$. Powder metallurgy methods, such as laser and spark plasma sintering of nickel-titanium

*corresponding author; e-mail: bulatgnmail@gmail.com powders, are mainly used for the synthesis of porous NiTi alloy with a crystalline structure [15-17]. Another method of porous NiTi production is generation of the bubble-like cells in the melt at a temperature above the melting (liquidus) temperature with subsequent vitrification of this melt $[8,18]$. Upon rapid solidification of the melt, these bubbles form a porous structure of the amorphous material. This method allows one to obtain a porous alloy with low degree of porosity at relatively low applied cooling rates. However, this method does not allow generation of nanosized pores, and the method does not exclude the appearance of crystalline inclusions in an amorphous matrix. Moreover, there is no clear understanding of the effect of a melt cooling protocol on pore morphology in porous NiTi alloy.

In the present work, based on atomistic dynamics simulations we show that the morphology of the pores in amorphous porous NiTi alloy can be controlled by means of applied cooling rates.

\section{Parameters of considered system}

The interaction energy between the atoms of titanium nickelide is defined through the modified embedded-atom method (MEAM) interatomic potential. The potential was proposed by Ko et al. for NiTi-based alloys [19, 20] and this type of potential correctly reproduces the structural and dynamic properties of NiTi alloy at low temperatures. The simulation cell contained 109744 atoms (54872 atoms of $\mathrm{Ni}$ and 54872 atoms of Ti). The periodic boundary conditions were applied in all the directions. Temperature and pressure were controlled by the Nose-Hoover thermostat and barostat with the damping parameters $Q_{T}=100 \Delta t$ and $Q_{P}=5000 \Delta t$, respectively. Here the time step was $\Delta t=1 \mathrm{fs}$. Initially, the simulated system was prepared so that it corresponded to the 

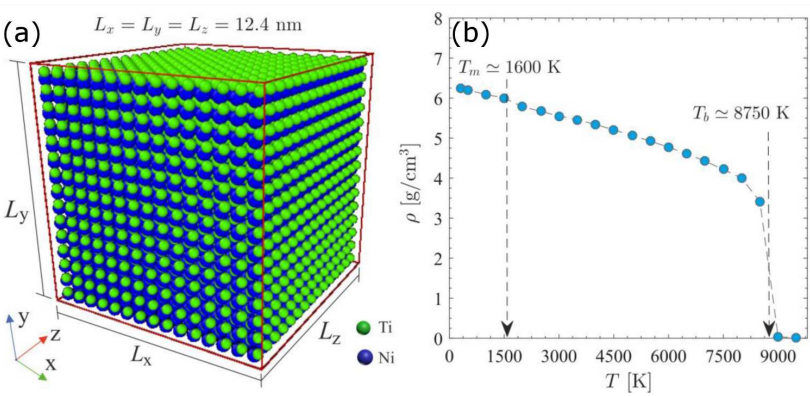

Fig. 1. (a) Fragment of the $B 2$ crystal structure for titanium nickelide with 6859 atoms. (b) Density of the system $\rho$ as function of temperature $T$ for equilibrium NiTi melt evaluated from atomistic dynamics simulations.

cubic $B 2$ crystalline phase at the temperature $T=0 \mathrm{~K}$ (see Fig. 1a). Then, this crystalline system was rapidly heated and equilibrated at the $(\mathrm{p}, \mathrm{T})$-states with pressure of $p=1 \mathrm{~atm}$ and with various temperatures in the range $T \in[300,9500] \mathrm{K}$.

The mass density of NiTi, evaluated from simulation data, as a function of temperature is shown in Fig. 1b. The density of the system decreases rapidly at temperatures above $8000 \mathrm{~K}$, and the "liquid-vapor" phase transition is observed at temperature $T_{b} \approx 8750 \mathrm{~K}$. We start the cooling procedure of a melt with the lowest density and this procedure allows to generate a porous amorphous solid. The equilibrium liquid sample NiTi at temperature $T=8500 \mathrm{~K}$ and density $\rho=3.44 \mathrm{~g} / \mathrm{cm}^{3}$ is the most suitable for this. Three samples of the porous amorphous NiTi alloy at temperature $T=300 \mathrm{~K}$ are obtained by rapid isobaric cooling of the liquid sample with the cooling rates $\vartheta_{c}=5 \times 10^{13}, 10^{14}$, and $5 \times 10^{14} \mathrm{~K} / \mathrm{s}$. Note that such very high cooling rates were recently obtained experimentally for nanometer-sized samples [21].

\section{Pore morphology under ultrafast cooling}

Figure 2a shows the snapshots of amorphous NiTi alloy with a nanoporous structure obtained by cooling of the melt at three different rates. The supercooling level of the system is $\left(T_{m}-T\right) / T_{m} \approx 0.8$, and the porous amorphous phase is stable at such deep supercooling level (see Fig. 2a). Remarkable feature of vitrification by ultrafast cooling is that the system does not have enough time during this cooling to form a dense homogeneous structure due to slow and uneven thermal compression [22, 23]. As a result, during a rapid isobaric cooling, pores form in the system.

The coefficient of porosity of the system is estimated using the relation [24]:

$$
\phi=\left(1-\frac{\rho}{\rho_{0}}\right) \times 100 \% .
$$

Here $\rho$ is the mass density of porous amorphous NiTi alloy, $\rho_{0}=6.24 \mathrm{~g} / \mathrm{cm}^{3}$ is the mass density of homogeneous amorphous NiTi alloy at temperature $T=300 \mathrm{~K}$.
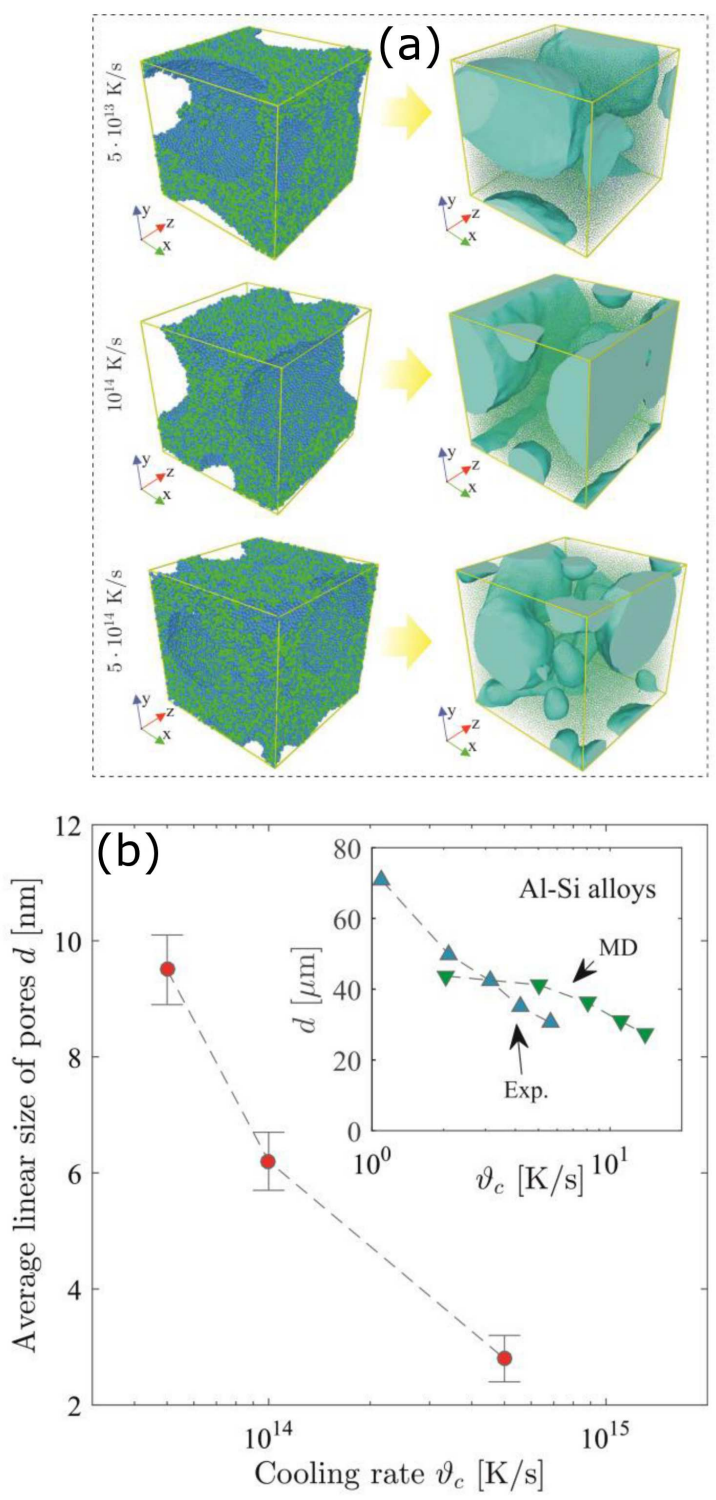

Fig. 2. (a) 3D-snapshots of amorphous NiTi alloy with porous structure at temperature $300 \mathrm{~K}$ (left column) and 3D-maps of the inside surface of the pores obtained at different cooling rates (right column). Green and blue spheres are the $\mathrm{Ti}$ and $\mathrm{Ni}$ atoms, respectively. (b) Average linear size of pores $d$ as function of the cooling rate $\vartheta_{c}$. Inset: experimental and simulation $d$ vs. $\vartheta_{c}$ data obtained for Al-based alloys [28, 29].

The parameter $\phi$ can take values from $0 \%$ to $100 \%$. So, one has $\phi=0 \%$ for a sample without pores, and $\phi=100 \%$ for the case of vacuum. We find that the mass density of NiTi melt increases from $\rho=3.44 \mathrm{~g} / \mathrm{cm}^{3}$ to $\rho=(4.3 \pm 0.15) \mathrm{g} / \mathrm{cm}^{3}$ during the applied quench procedure from the state with the temperature $8500 \mathrm{~K}$ to the solid state with the temperature $300 \mathrm{~K}$. The generated porous amorphous alloy has the porosity $\phi=(31 \pm 3) \%$ and value of the coefficient $\phi$ is independent of cooling at such high rates. Note that materials with such level of porosity are widely used in production of membranes 
and electrodes for energy storage elements, where the cell structures with meso- and microporosity allow one to achieve optimal electrochemical characteristics of the materials [25-27].

In Fig. 2a the constructed maps of the inside surface of the pores for all three samples are shown. As seen from Fig. 2a, the porous structure of the amorphous alloy appears due to the empty cells of spherical shape uniformly distributed throughout the system. Linear sizes of these cells range from $1 \mathrm{~nm}$ to $10 \mathrm{~nm}$. It is important to note that there is correlation between the cell concentration and the cooling rate, which was taken in the cooling protocol to generate the porous amorphous alloy. The pores coalesce and form hollow ramified tunnels that permeate the whole system at vitrification for cooling rates more than $10^{14} \mathrm{~K} / \mathrm{s}$. At cooling rates less than $10^{14} \mathrm{~K} / \mathrm{s}$, lower concentration of pores is observed in the amorphous sample, where the pores are mainly isolated. This is clearly seen in Fig. 2a, where the geometry of the pores is shown for the case of the porous amorphous alloy generated by cooling at the rate of $5 \times 10^{13} \mathrm{~K} / \mathrm{s}$.

We find that the average linear size $d$ of pores decreases rapidly with increasing cooling rate $\vartheta_{c}$, whereas the porosity of the system remains constant. This trend is also valid for other types of systems. For example, as seen in the inset to Fig. 2b, the experimental and simulation data for the $\vartheta_{c}$-dependence of the linear size of pores $d$ obtained for Al-based alloys show similar trend [28, 29]. The presence of such dependence between the quantities $\vartheta_{c}$ and $d$ is explained by the fact that more pores with smaller sizes are formed at relatively higher cooling rate. The growth of such small pores leads to the formation of percolated pores. For example, at the cooling rate of $5 \times 10^{14} \mathrm{~K} / \mathrm{s}$, the pores have a high concentration and these pores do not have time to completely coalesce. As a result, the percolating pores are formed. For comparison, at the cooling rate of $5 \times 10^{13} \mathrm{~K} / \mathrm{s}$, the pores coalesce and form large spherical cavities (see Fig. 2a).

\section{Concluding remarks}

In the present work, we have shown that the porous amorphous NiTi alloy can be generated by ultrafast cooling of a low-density melt and these results can be useful in the development of designing methods of porous amorphous alloys with the necessary parameters of the porous structure. The main results of this study can be summarized as follows:

1. The equation of state, $\rho(T)$, of the equilibrium $\mathrm{NiTi}$ melt for the temperature ranging from $T=300 \mathrm{~K}$ to $9500 \mathrm{~K}$ was determined using isobaric atomistic dynamics simulation with MEAM potential, proposed by Ko et al.

2. Amorphous NiTi alloy with isolated pores and percolated porous structures can be generated by means of ultrafast isobaric cooling of low-density liquid melt, and the generated amorphous samples are characterized by the porosity $\phi=(31 \pm 3) \%$.
3. It was shown that the average linear size of pores depends on magnitude of the cooling rate, by means of which the amorphous alloy was generated. This result is in qualitative agreement with experimental and simulation data obtained for other types of porous alloys.

\section{Acknowledgments}

This work is supported by the Russian Science Foundation (project No. 19-12-00022). Atomistic dynamics simulations were performed by using the computational cluster of Kazan Federal University.

\section{References}

[1] J.W. Mwangi, L.T. Nguyen, V.D. Bui, T. Berger, H. Zeidler, A. Schubert, J. Manuf. Process. 38, 355 (2019).

[2] S.A. Shabalovskaya, Int. Mater. Rev. 46, 233 (2001).

[3] Y. Qiu, H. Yu, M.L. Young, Shape Mem. Superelast. 1, 479 (2015).

[4] R.M. Khusnutdinoff, Acta Phys. Pol. A 129, 293 (2016).

[5] K. Otsuka, X. Ren, Prog. Mater. Sci. 50, 511 (2005).

[6] N. Levintant-Zayonts, L. Kwiatkowski, Z. Swiatek, J. Brzozowska, Acta Phys. Pol. A 132, 210 (2017).

[7] P. Ternik, M. Zadravec, M. Svetec, R. Rudolf, Acta Phys. Pol. A 128, 43 (2015).

[8] J. Schroers, C. Veazey, M.D. Demetriou, W.L. Johnson, J. Appl. Phys. 96, 7723 (2004).

[9] M.A. Qidwai, P.B. Entchev, D.C. Lagoudas, V.G. DeGiorgi, Int. J. Solid Struct. 38, 8653 (2001).

[10] G. Ryan, A. Pandit, D.P. Apatsidis, Biomaterials 27, 2651 (2006).

[11] A. Bansiddhi, T.D. Sargeant, S.I. Stupp, D.C. Dunand, Acta Biomater. 4, 773 (2008).

[12] G. Sneddon, A. Greenaway, H.H.P. Yiu, Adv. Energy Mater. 4, 1301873 (2014).

[13] T. Fujita, Sci. Technol. Adv. Mater. 18, 724 (2017).

[14] A.E. Evans, J.W. Hutchinson, M.F. Ashby, Prog. Mater. Sci. 43, 171 (1999).

[15] D.V. Dudina, B.B. Bokhonov, E.A. Olevsky, Materials 12, 541 (2019).

[16] I. Shishkovsky, I. Yadroitsev, I. Smurov, Phys. Proced. 39, 447 (2012).

[17] P.S. Liu, G.F. Chen, Porous Materials Processing and Applications Oxford (UK) 2014.

[18] J. Schroers, C. Veazey, W.L. Johnson, Appl. Phys. Lett. 82, 370 (2003).

[19] W.-S. Ko, B. Grabowski, J. Neugebauer, Phys. Rev. B 92, 134107 (2015).

[20] Z.-Y. Zeng, C.-E Hu, L.-C. Cai, X.-R. Chen, F.-Q. Jing, J. Appl. Phys. 109, 043503 (2011).

[21] L. Zhong, J. Wang, H. Sheng, Z. Zhang, S.X. Mao, Nature 512, 177 (2014). 
[22] B.N. Galimzyanov, V.I. Ladyanov, A.V. Mokshin, J. Cryst. Growth 526, 125214 (2019).

[23] A.V. Mokshin, B.N. Galimzyanov, D.T. Yarullin, JETP Lett. 110, 511 (2019).

[24] F.A.L. Dullien, Porous Media. Fluid Transport and Pore Structure, Academic Press, 1992.

[25] M. Singh, J. Kaiser, H. Hahn, Batteries 2, 35 (2016).

[26] D. Qu, AIP Conf. Proc. 1597, 14 (2014).
[27] J. Song, J. Kim, T. Kang, D. Kim, Sci. Rep. 7, 42521 (2017).

[28] V.A. Hosseini, S.G. Shabestari, R. Gholizadeh, Mater. Des. 50, 7 (2013).

[29] T. Wang, D. An, Q. Zhang, T. Dai, M. Zhu, IOP Conf. Series Mater. Sci. Eng. 84, 012046 (2015). 